\title{
THE KNEE
}

\author{
P Sanville, D A Nicholson, P A Driscoll
}

Most knee injuries are confined to the soft

The exposed position of the knee and its functional demands make it one of the most vulnerable joints to injury, especially in athletes. In most cases, however, the plain films look normal and clinical examination does not show any disruption of ligaments. This article describes the common types of bony injury that are found and advises how to assess plain radiographs.

\section{Important anatomical considerations}
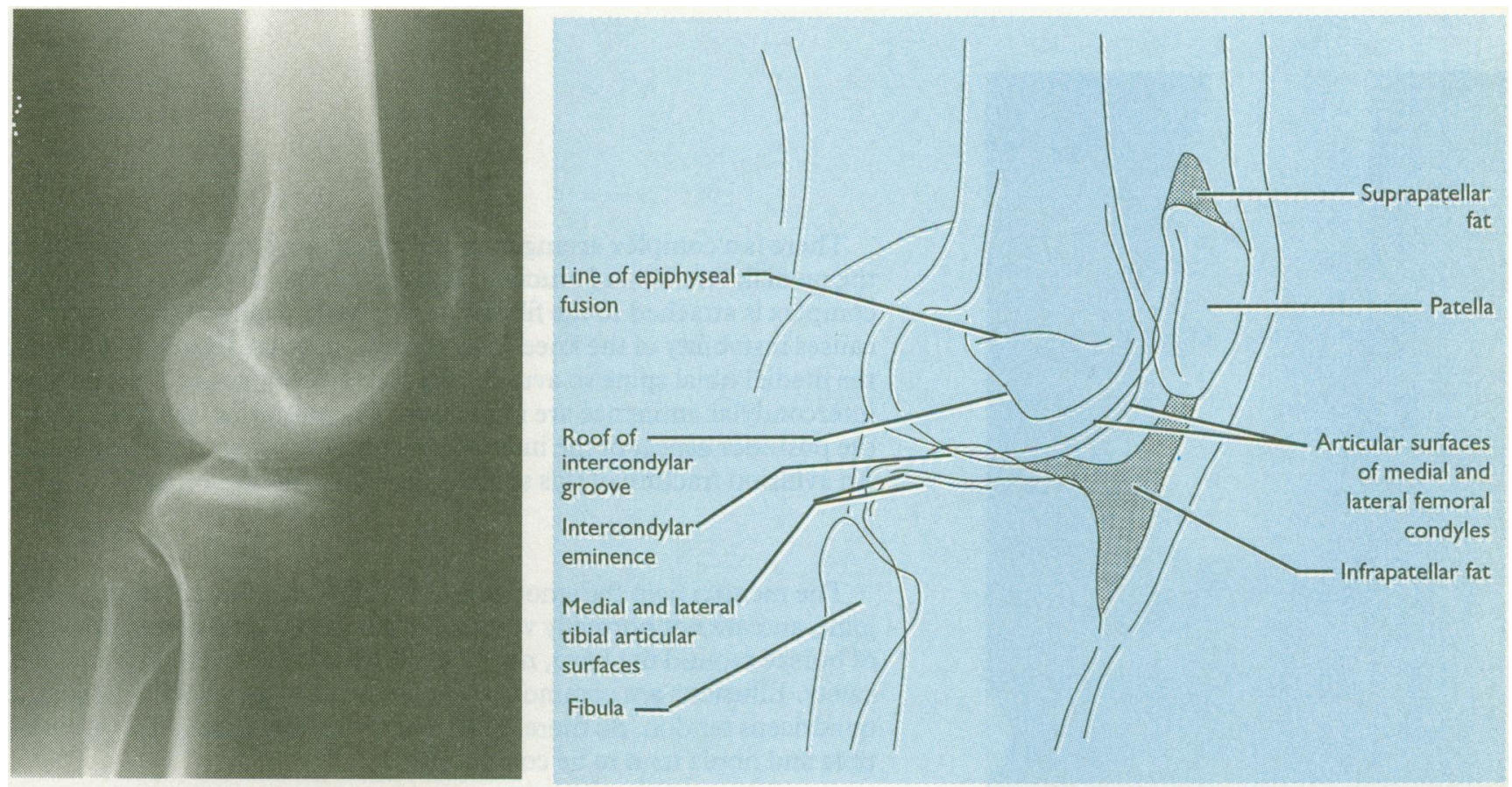

FIG 1-Lateral radiograph and line diagram of knee

Fractures of the fibular neck and head may be associated with damage to the common peroneal nerve and collateral ligament complex and may be part of a pronationlateral rotation ankle injury.

The arterial blood supply can be compromised in fractures near the adductor canal and popliteal fossa because of impingement of fracture fragments. Tibial and fibular fractures can cause compression of vessels because of haematoma in the leg compartments.

\section{Adult}

The knee is a synovial joint formed by the femoral condyles articulating with the tibial condyles (figs 1 and 2). The patella lies within the quadriceps tendon. The posterior surface of this sesamoid bone has a steep sloping medial articulating facet and a shallower lateral facet for articulation with the femoral condyles.

The common peroneal nerve runs close to the neck of the fibula and is prone to injury. Posteriorly, the popliteal artery is closely related to the tibial plateau and may be damaged by fracture fragments or in dislocation of the knee. 

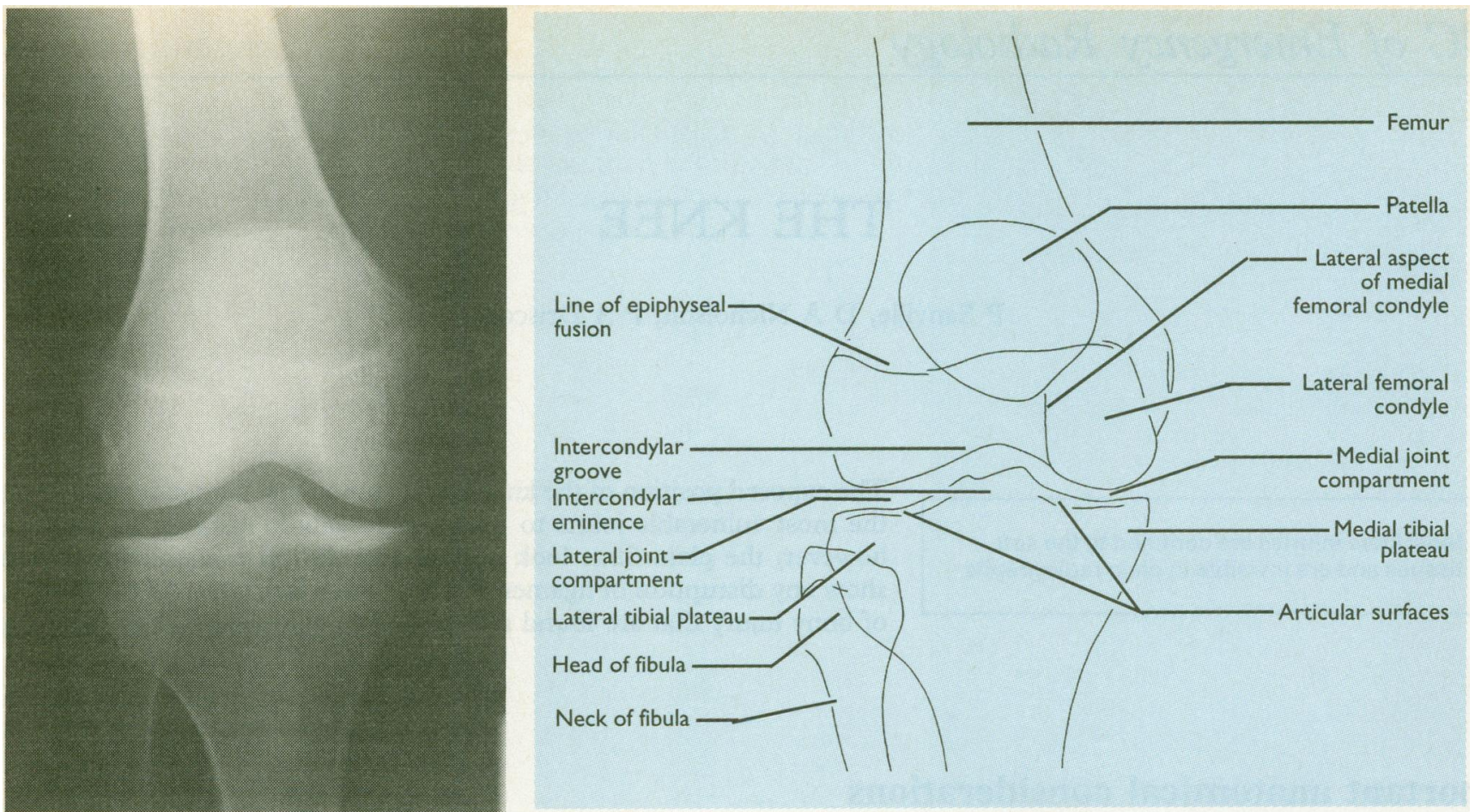

FIG 2-Anteroposterior radiograph and line diagram. Note the slight reduction of the medial joint compartment.

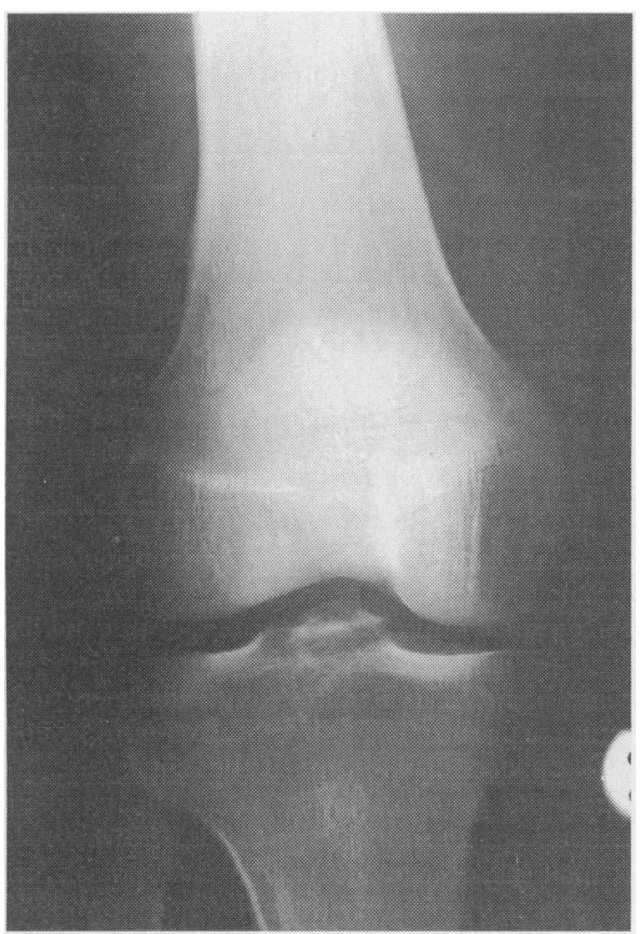

FIG 3-Avulsion fracture of the intercondylar eminence.
There is a complex arrangement of ligaments around the knee including the medial, lateral, and cruciate ligaments. Part of the lateral ligament complex is attached to the fibular head. Injury to any of these complexes causes instability of the knee. The anterior cruciate ligament is attached to the medial tibial spine so avulsion fractures of this spine or of the base of the intercondylar eminence are important. The posterior cruciate attaches to the posterior aspect of the intercondylar region away from the lateral spine. An avulsion fracture of this spine is therefore less important.

The menisci help the smooth movement, including rotation of the knee joint, and are not normally visible in plain films. There are a large number of bursae around the knee, not all of which communicate with the joint cavity. Effusions are commonly seen in the suprapatellar bursa behind the quadriceps tendon. As there is little overlying soft tissue, fractures of the tibia and fibula tend to be compounded.

\section{Assessment of the physis}

Look carefully for widening or compression, which may affect part or all of the physis. Compression fractures can be very subtle. Injury to the physis can be associated with stunted growth.

\section{Children}

The distal femoral and proximal tibial epiphyses are present from birth until 18-20 years. The non-ossified cartilage overlying the femoral epiphysis is visible in the lateral film as an irregular soft tissue density around the epiphysis. The distal femoral epiphysis may be seen to be cleft.

The patella starts to ossify between the ages of 3 and 6 years. Several ossification centres may fail to fuse, simulating fractures. A horizontally bifid patella is a normal variant best seen in the lateral film, which usually goes on to unite. 


\section{Mechanisms of injury}

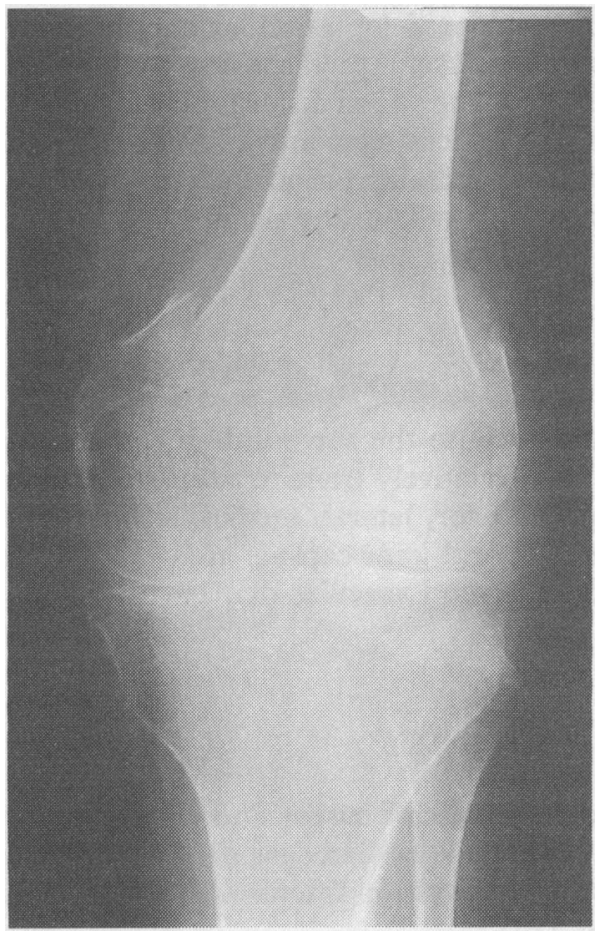

FIG 4-Impacted comminuted supracondylar fracture. Note the fracture lines extending up the femoral shaft.

\section{Femur-distal two thirds}

Fractures of the femoral shaft usually result from considerable force. The quadriceps and hamstring muscles tend to produce shortening and displacement of the fractures and there can be considerable loss of blood. Serious rotation of the distal fragment is easily overlooked unless the whole femur is surveyed in two projections.

Supracondylar fractures in adults often result in rotation of the distal fragment because of the pull of the gastrocnemius muscle. In children, these fractures are usually minimally displaced.

Femoral condylar fractures can be displaced or undisplaced and affect one or both condyles. If the fracture is completely intra-articular the bones may not reunite because the synovial fluid interferes with organisation of the haematoma. Severely comminuted condylar fractures may be associated with a spiral fracture of the distal femur.

\section{Tibia and fibula-proximal third}

A fracture of the lateral tibial condyle commonly results from severe valgus stress (such as being hit by a car bumper). With further force the fibular neck fractures and the medial collateral and cruciate ligaments tear causing medial subluxation of the tibia. Bicondylar fractures may be seen. If there is a severely depressed fracture of the tibial plateau and no fibular fracture then disruption of the proximal tibiofibular joint has occurred.
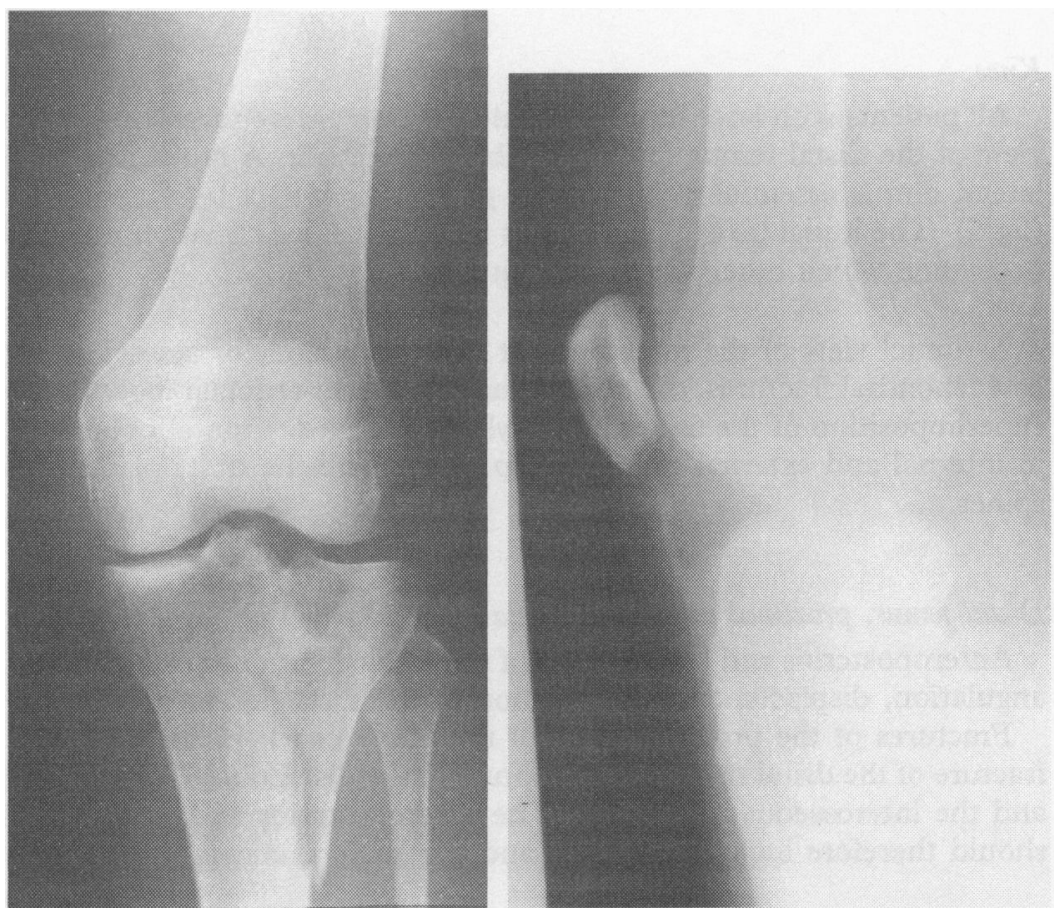

FIG 5-Anteroposterior (left) and horizontal beam lateral (right) films of tibial plateau fracture due to valgus strain. The large lipohaemarthrosis is due to a comminuted, displaced fracture of the lateral tibial plateau affecting the intercondylar eminence. Note that the fibular head is intact implying disruption of the tibiofibular ligament.
Isolated silent fractures of the proximal fibula are seen in patients who have had parachute accidents. These are often misdiagnosed as a soft tissue injury clinically.

Osgood-Schlatter disease is due to recurrent contraction of the quadriceps in teenage athletes. Plain radiographs may show soft tissue swelling over the tibial tubercle, which is raised away from the tibia. These findings are non-specific and the disease should be diagnosed only in conjunction with the clinical findings of pain, tenderness, and swelling.

\section{Patella}

When the patella is fractured by direct violence a femoral shaft fracture and posterior dislocation of the hip may also occur. This is seen typically in patients who hit the dashboard in car accidents.

\section{Factors predisposing to patellar dislocation}

- Hypoplastic lateral femoral condyle

- Genu valgum

- High lying patella (patella alta)

- Abnormal insertion of vastus lateralis
Fractures of the patella may be vertical, horizontal, or comminuted. Vertical fractures are usually stable but distraction of the fragments is common in horizontal fractures. If the bones are undisplaced in the initial film, weekly follow up for three weeks is advisable to detect late separation. 


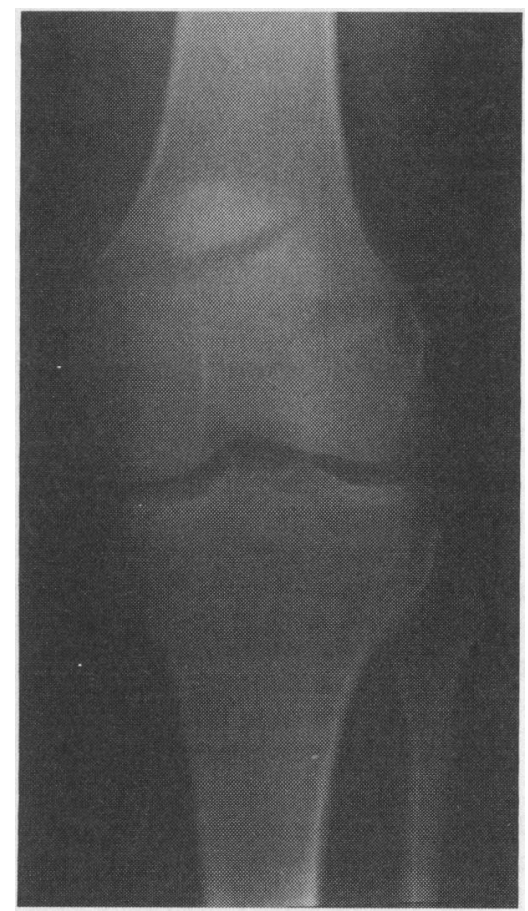

FIG 6-Anteroposterior film of horizontal patellar fracture showing slight separation of the fragments. Late separation may occur.

\section{Types of view}

\author{
Radiographic views of the knee \\ Standard: \\ Anteroposterior \\ Lateral \\ Additional: \\ Skyline patellar and tunnel views \\ Oblique \\ Anteroposterior with valgus and varus \\ stress
}

\section{Lipohaemarthrosis}

This is seen when the blood and fat that has leaked out of the medullary cavity, typically in a tibial plateau fracture, forms a fat-blood fluid level in the suprapatellar pouch. This may be the only sign of such a fracture on plain radiography. The $x$ ray beam must be tangential to this fluid-fluid layer-that is, horizontal. A vertical beam would superimpose the two layers.
Dislocation of the patella often recurs. It is seen typically in teenage girls and always occurs laterally because of the flat lateral condyle and the oblique pull of the quadriceps muscle. It is caused by muscular contraction or a blow to the medial patella. There are several predisposing conditions that can be assessed in plain films. In complete lateral dislocation the patella will lie lateral and parallel to the lateral femoral epicondyle. Minor degrees of subluxation can be seen. An osteochondral avulsion fracture from the lateral femoral condyle or the medial patella in the skyline view suggests a previous patellar dislocation.

\section{Dislocation of the knee}

Dislocation of the knee is rare because the surrounding ligments are strong. However, it can occur with relatively trivial trauma. The tibia is usually displaced anteriorly but posterior, lateral, medial, and rotational dislocation can occur. The ligament and joint capsule are always severely damaged and proximal tibial fracture and vascular damage are often present.

\section{Soft tissue injuries}

Most knee injuries are confined to the soft tissues and are not visible in plain radiographs. A varus stress to the knee damages the lateral ligament complex. There may be an associated avulsion fracture of the head of the fibula or the proximal tibia (fig 7).

\section{Knee}

All patients with knee injuries should have an anteroposterior and lateral view of the distal femur, proximal tibia, and fibula. A horizontal beam lateral film is essential in all trauma patients to detect lipohaemarthrosis (fig 5). The initial plain film findings and clinical examination are used to determine which other views are required.

A tunnel view of the intercondylar notch is useful for assessing osteochondral fractures, the tibial spines, and intra-articular loose bodies as superimposition of the femoral condyles is reduced. Frontal oblique views in internal and external rotation help to evaluate the tibial plateau and spines.

\section{Distal femur, proximal tibia, and fibula}

Anteroposterior and lateral views of the whole femur are needed to assess angulation, displacement, and rotation of the distal femur accurately.

Fractures of the proximal third of the fibula can be associated with a fracture of the distal tibia or with rupture of the distal tibiofibular ligaments and the interosseous membrane. The anteroposterior and lateral views should therefore include the knee and the ankle if clinically indicated.

\section{System of radiological assessment}

\section{The medullary bone}

Look for subtle lucent fracture lines or discontinuity in the trabecular pattern. Stress fractures of the proximal tibia appear as a thickened sclerotic transverse band and a lucent line may extend through part of the cortex.

\section{Lateral projection}

Follow the ABCs system of assessment.

Check the adequacy and quality of the radiograph-The fibular head overlaps the posterior tibia and the femoral condyles are superimposed. The radiographic density should allow the soft tissues, and particularly the suprapatellar pouch, to be evaluated.

Check alignment of bones-With the knee extended the patella lies anterior to the proximal portion of the femoral condyles. The patella may be invisible if there is complete lateral dislocation as it becomes superimposed over the lateral femoral condyle. The femoral condyles should articulate with the tibial plateau. 


\section{Subcutaneous emphysema}

In perforating injuries or compound fractures air in the soft tissues of joint spaces can cause areas of increased lucency.

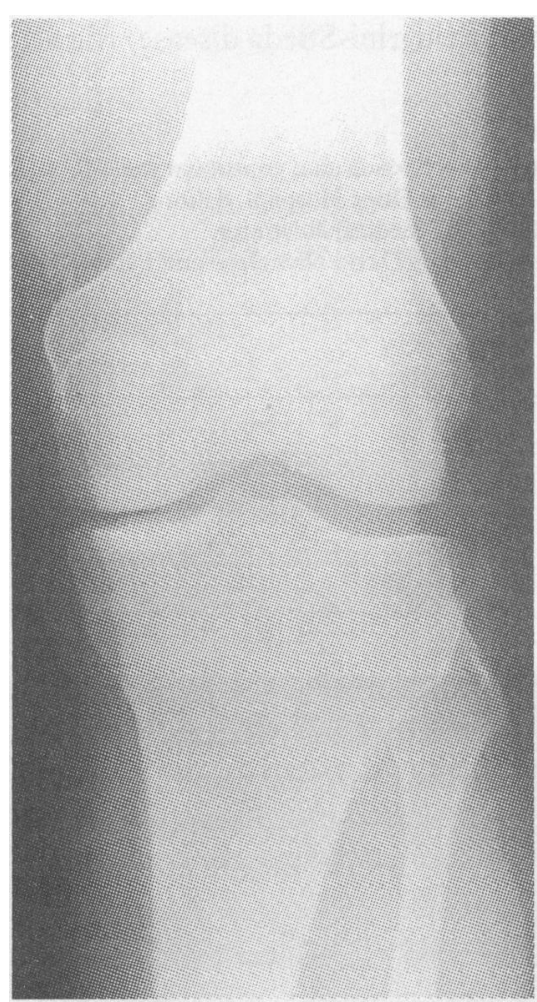

FIG 7-Anteroposterior film of varus strain injury showing lateral widening of the joint space and an avulsion fracture of the lateral tibial condyle.
Check bone margins and density-Starting with the distal femur, trace around the cortical margin ensuring that there is no discontinuity, particularly in the supracondylar area. Trace the tibial cortex checking for a minimally displaced plateau fracture. This will appear either as an area of local sclerosis due to impaction or as a step defect. Trace the margin of the patella. Vertical fractures of the patella are usually undisplaced and invisible in the lateral film. Horizontal patellar fractures should be visible.

Cartilage and joints-Meniscal tears are not visible in plain radiographs but an associated effusion may be seen.

Check soft tissues-An area of increased lucency due to fat is usually seen behind the quadriceps and prepatellar tendon. Joint effusions in the prepatellar pouch appear as a tongue of soft tissue density extending superiorly from the patellar-femoral joint into this lucent area. The patella will be displaced anteriorly and tilted inferiorly by a large effusion.

\section{Anteroposterior projection}

Check the adequacy and quality of the radiograph-The film should be centred on the joint space and the lateral tibia and the head of the fibula should overlap slightly. The patella should just be visible in the midline projected over the intercondylar notch.

Check alignment of bones-The femoral condyles should sit on the tibial condyles. The patella should lie in the midline.

Check bone margins and density-Starting with the most proximal part of the femur, trace the cortical margin clockwise. Repeat this for the tibia, paying particular attention to the tibial plateau. Check that the medial and lateral tibial spines are intact. Look for small avulsion fractures at the site of insertion of the medial and lateral collateral ligaments. Observe the neck of the fibula for a fracture. Examine the patella.

Cartilage and joints-Assess the joint spaces between the femoral and tibial condyles. The medial and lateral compartments should be equal in height. Note the double contour of the medial tibial plateau-the distal line is the articular surface.

Check soft tissues-Look for evidence of subcutaneous emphysema or any foreign body. Chondrocalcinosis due to calcification of the menisci produces a thin line of calcification parallel to the tibial condyles, especially in older patients with osteoarthritis.

\section{Catches to avoid}

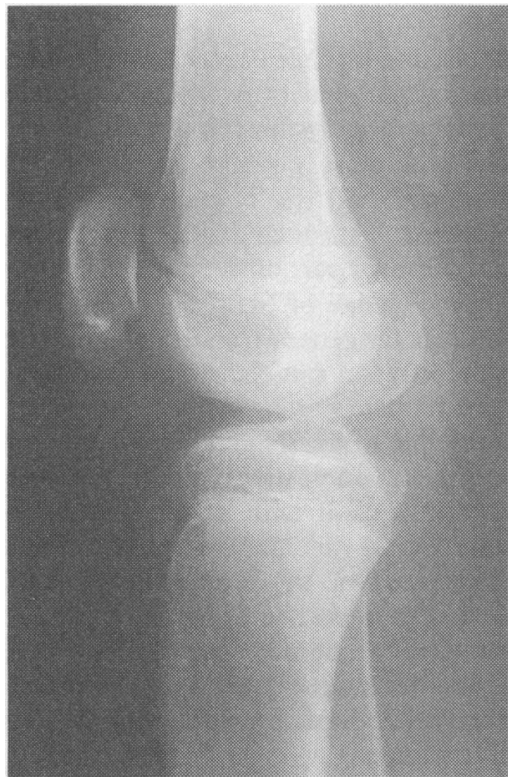

FIG 8-Well defined sclerotic margins between the patella and an accessory ossification centre in a 12 year old child. Note the normal epiphyses and tibial tubercle apophysis.

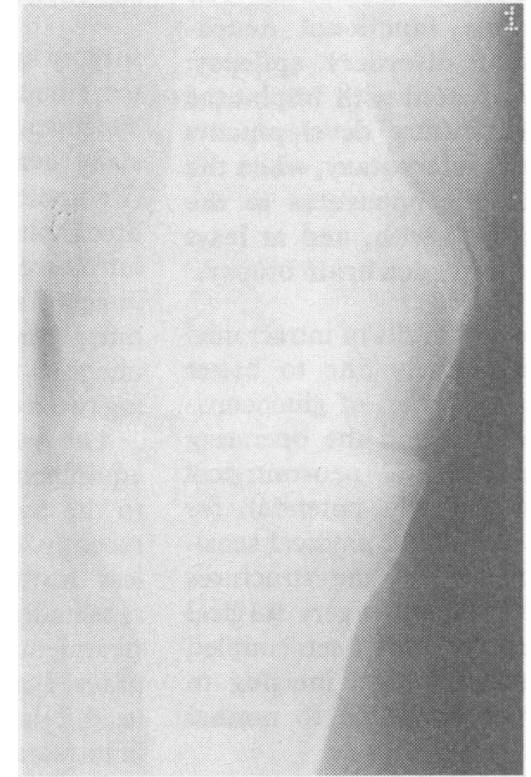

FIG 9-Pelligrini-Stieda disease. This should be distinguished from an acute avulsion injury.
The fabella is commonly seen lying in the lateral head of gastrocnemius, posterolateral to the knee.

The normal fat pad lying immediately posterior to the patellar tendon may look like a small lipohaemarthrosis in the lateral view but it will be visible in a film taken with a vertical $x$ ray beam, unlike a true lipohaemarthrosis.

The patella may be bipartite. In this case a separate upper outer portion with well defined rounded margins is seen in the anteroposterior and skyline patellar views. Less commonly the patella is segmented in up to four pieces.

In children the anteroposterior view may show developmental lucent stripes on the medial aspect of the distal femoral epiphysis which simulate fractures. 


\title{
Summary
}

\section{Diagnostic quality}

Alignment of bones

Femur: tibia

Patella

Bone margins and density

Femur, tibia, patella, fibula

Cartilage and joints

Femorotibial

Patellar-femoral
Soft tissues

Effusions

Lipohaemarthrosis

Chondrocalcinosis

Surgical emphysema

Foreign bodies
An ununited accessory ossification centre of the fibular head will have a smooth corticated margin unlike a fracture. A thin line of ossification may also be seen in the medial collateral ligament next to the insertion point on the medial femoral epicondyle. This is caused by an old avulsion injury of this ligament with subsequent calcification within the subperiosteal haematoma (Pelligrini-Stieda disease) (fig 9).

P Sanville is Cook research fellow in radiology, D A Nicholson is consultant radiologist, and P A Driscoll is senior lecturer in emergency medicine at Hope Hospital, Salford.

The line drawings were prepared by Mary Harrison, medical illustrator.

The ABC of Emergency Radiology has been edited by David Nicholson and Peter Driscoll.

\section{Minimally Invasive Surgery}

\section{Neurosurgery}

\author{
D G T Thomas, N D Kitchen
}

This is the seventh in a series of articles describing the current techniques in minimal access surgery. The articles have been written to inform non-specialists of developments in this rapidly moving area.

\section{Gough-Cooper}

Department of

Neurological Surgery,

Institute of Neurology,

London WC1N 3BG

D G T Thomas, professor of

neurosurgery

N D Kitchen, clinical

research fellow

Correspondence to:

Dr Kitchen.

$B M \widetilde{f} 1994 ; 308: 126-8$
The introduction of minimally invasive techniques has greatly improved results for intracranial neurosurgery. Stereotaxy and improved imaging techniques have reduced surgical trauma by allowing surgeons to plan the least damaging route to operative sites and by increasing surgical precision. Stereotaxy has also allowed brain biopsies to be taken from sites such as the brain stem, which were rarely sampled before because free hand biopsy was so dangerous. Brain tumours can now be treated by interstitial radiotherapy-stereotactic insertion of catheters into the lesion for loading of radioactive iodine -or radiosurgery-focusing of intense beams of radiation on lesions without needing surgical incisions. Endoscopic neurosurgery can be used to reach cavities such as the ventricular system or cystic tumours. With interventional neuroradiology fine catheters can be introduced into most vessels in the cranium for embolisation or dilatation. The development of augmentative functional neurosurgery means that movement disorders, epilepsy, and intractable pain can be treated with implanted neurostimulating electrodes. Future developments will probably include frameless stereotaxy, when the rigid attachment of stereotactic apparatus to the patient's head can be dispensed with, and at least partial automation of procedures such brain biopsy.

During the last three decades the results of intracranial neurosurgery have improved, largely due to better neuroanaesthesia and the introduction of glucocorticoids to control cerebral oedema and the operating microscope. Most major intracranial neurosurgical procedures, however, have a high potential for morbidity and mortality because of the physical sensitivity and neurological eloquence of the structures involved. In minimally invasive neurosurgery surgical techniques, particularly stereotaxy, have been coupled with improvements in neuroradiological imaging to increase precision and to reduce trauma to normal tissues.

\section{Stereotaxy}

Stereotaxy was introduced by Horsley and Clarke at the beginning of this century for experimental brain

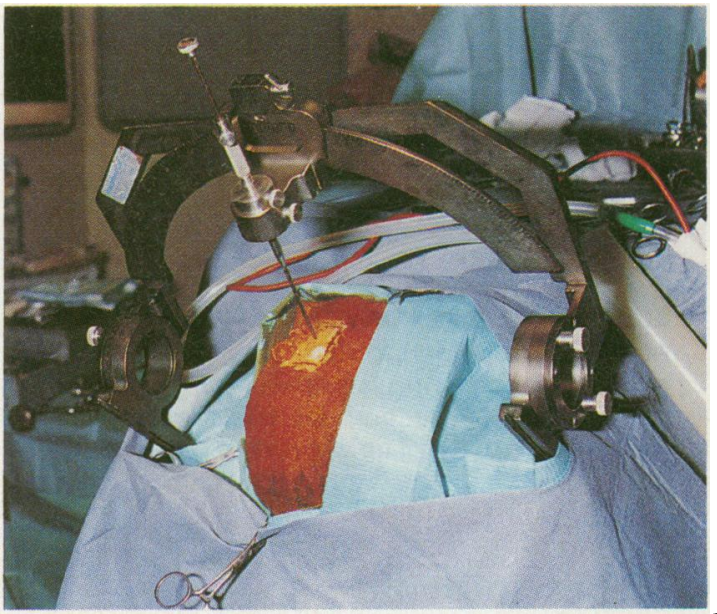

FIG $1-$ Stereotactic frame in use during craniotomy. Entry point and trajectory to target have been chosen

surgery in animals and has been successfully developed for clinical use in humans over the past 40 years. ${ }^{1}$ Technically satisfactory systems are now commercially available, the most popular being the CRW (Radionics, Boston, USA) (fig 1) and Leksell (Elekta, Stockholm, Sweden). With such devices a probe can be introduced into a known part of the brain identified by imaging and a knowledge of brain anatomy. A computer can be used to generate a three dimensional image so that the surgeon can choose the least damaging route to the operative site.

The basic principle of nearly all current stereotactic equipment is firm fixation of the stereotactic apparatus to the patient's skull vault with metal pins or, more recently, dental fixation (fig 2). The second method is less invasive and allows the frame to be applied repeatedly so that stereotactic imaging, planning of treatment, and surgery may be carried out at different times. For stereotactic surgery local anaesthesia can be used, but general anaesthesia provides optimal control of intracranial conditions.

\section{Stereotactic biopsy and craniotomy}

In stereotactic brain biopsy a fine cannula just over $1 \mathrm{~mm}$ in diameter is passed down into the radiographic- 